\title{
Uniformly Stable Positive Monotonic Solution of a Nonlocal Cauchy Problem
}

\author{
A. M. A. El-Sayed ${ }^{1}$, E. M. Hamdallah ${ }^{1}$, Kh. W. Elkadeky ${ }^{2}$ \\ ${ }^{1}$ Faculty of Science, Alexandria University, Alexandria, Egypt \\ ${ }^{2}$ Faculty of Science, Garyounis University, Benghazi, Libya \\ Email: \{amasayed, emanhamdalla\}@hotmail.com,k-welkadeky@yahoo.com
}

Received October 9, 2011; revised December 7, 2011; accepted December 30, 2011

\begin{abstract}
In this paper, we study the existence of a uniformly stable positive monotonic solution for the nonlocal Cauchy problem $x^{\prime}(t)=f(t, x(t)), t \in[0, T]$ with the nonlocal condition $\sum_{j=1}^{m} b_{j} x\left(\eta_{j}\right)=x_{1}$, where $\eta_{j} \in(0, a) \subset[0, T]$.
\end{abstract}

Keywords: Nonlocal Cauchy Problem; Local and Global Existence Nondecreasing Positive Solution; Continuous Dependence; Lyapunov Uniformly Stability

\section{Introduction}

Problems with non-local conditions have been extensively studied by several authors in the last two decades. The reader is referred to (see [1-14] and [15-18]) and references therein.

Here we are concerned with the nonlocal Cauchy problem

$$
\begin{gathered}
x^{\prime}(t)=f(t, x(t)), t \in[0, T], \\
\sum_{j=1}^{m} b_{j} x\left(\eta_{j}\right)=x_{1}, \eta_{j} \in(0, a) \subset[0, T], \text { and }\left(\sum_{j=1}^{m} b_{j}\right) \neq 0 .
\end{gathered}
$$

Let $X$ be the class of all continuous functions defined on $[0, T], T<\infty$ with the norm

$$
\|x\|=\sup _{t \in[0, T]}|x(t)|, x \in X .
$$

Let $Y$ be the class of all continuous functions defined on $\left[t_{0}, T\right], T<\infty$ with the equivalent norm

$$
\|x\|=\sup _{t \in[0, T]} e^{-N\left(t-t_{0}\right)}|x(t)|, x \in Y,
$$

where $t_{0}=\max \left\{\eta_{j}, j=1,2, \cdots, m\right\}$, and $N$ is positive arbitrary.

Here we firstly study, in $X$, the local existence of the solution of the problem (1)-(2) and the continuous dependence of the parameter $x$, will be proved.

Secondly, we study, in $Y$, the global existence and Lyapunov uniform stability of the solution of the problem (1)-(2).

\section{Integral Equation Representation}

Consider the nonlocal Cauchy problem (1)-(2).

Let $f:[0, T] \times R^{+} \rightarrow R^{+}$is continuous and satisfies the Lipschitz condition

$$
\begin{aligned}
& |f(t, x)-f(t, y)| \leq k|x-y|, k>0, \\
& \text { for all } x, y \in R^{+}
\end{aligned}
$$

Lemma 2.1. The solution of the nonlocal Cauchy problem (1)-(2) can be expressed by the integral equation

$$
x(t)=B\left(x_{1}-\sum_{j=1}^{m} b_{j} \int_{0}^{\eta_{j}} f(s, x(s)) \mathrm{d} s\right)+\int_{0}^{t} f(s, x(s)), \mathrm{d} s
$$

where $B=\left(\sum_{j=1}^{m} b_{j}\right)^{-1}$.

Proof. Integrating the Equation (1), we obtain

$$
x(t)=x(0)+\int_{0}^{t} f(s, x(s)) d s .
$$

Let $t=\eta_{j}$ in (5), we obtain

$$
x\left(\eta_{j}\right)=x(0)+\int_{0}^{\eta_{j}} f(s, x(s)) \mathrm{d} s,
$$

and

$$
\sum_{j=1}^{m} b_{j} x\left(\eta_{j}\right)=\sum_{j=1}^{m} b_{j} x(0)+\sum_{j=1}^{m} b_{j} \int_{0}^{\eta_{j}} f(s, x(s)) \mathrm{d} s .
$$

Substitute from (2) into (7), we obtain

$$
x(0)=B\left(x_{1}-\sum_{j=1}^{m} b_{j} \int_{0}^{\eta_{j}} f(s, x(s)) \mathrm{d} s\right) .
$$


Substitute from (8) into (5), we obtain

$x(t)=B\left(x_{1}-\sum_{j=1}^{m} b_{j} \int_{0}^{\eta_{j}} f(s, x(s)) \mathrm{d} s\right)+\int_{0}^{t} f(s, x(s)) \mathrm{d} s$.

Corollary 2.1. The solution of the integral Equation (4) is nondecreasing.

Proof. Let $x$ be a solution of the integral Equation (4), then for $t_{1}<t_{2}$, we have

$$
\begin{aligned}
x\left(t_{1}\right) & =B\left\{x_{1}-\sum_{j=1}^{m} b_{j} \int_{0}^{\eta_{j}} f(s, x(s)) \mathrm{d} s\right\}+\int_{0}^{t_{1}} f(s, x(s)) \mathrm{d} s \\
& <B\left\{x_{1}-\sum_{j=1}^{m} b_{j} \int_{0}^{\eta_{j}} f(s, x(s)) \mathrm{d} s\right\}+\int_{0}^{t_{2}} f(s, x(s)) \mathrm{d} s \\
& =x\left(t_{2}\right),
\end{aligned}
$$

which proves that the solution $x$ of the integral Equation (4) is nondecreasing.

Corollary 2.2. Let $f$ be satisfies (3). The solution of the integral Equation (4) is positive for $t \in[a, T]$.

Proof. Let $x$ be a solution of the integral Equation (4), and $x_{1}>0$, for $t \in[a, T]$, we have

$$
\int_{0}^{\eta_{j}} f(s, x(s)) \mathrm{d} s \leq \int_{0}^{t} f(s, x(s)) \mathrm{d} s, \quad \eta_{j}<t
$$

and

$$
\begin{aligned}
T x(t)-T y(t) & =-B \sum_{j=1}^{m} b_{j} \int_{0}^{\eta_{j}} f(s, x(s)) \mathrm{d} s+\int_{0}^{t} f(s, x(s)) \mathrm{d} s+B \sum_{j=1}^{m} b_{j} \int_{0}^{\eta_{j}} f(s, y(s)) \mathrm{d} s-\int_{0}^{t} f(s, y(s)) \mathrm{d} s \\
= & -B \sum_{j=1}^{m} b_{j} \int_{0}^{\eta_{j}}\{f(s, x(s))-f(s, y(s))\} \mathrm{d} s+\int_{0}^{t}\{f(s, x(s))-f(s, y(s))\} \mathrm{d} s \\
|T x(t)-T y(t)| & \leq k|B| \sum_{j=1}^{m}\left|b_{j}\right| \int_{0}^{\eta_{j}}|x(s)-y(s)| \mathrm{d} s+k \int_{0}^{t}|x(s)-y(s)| \mathrm{d} s \\
& \leq k|B| \sum_{j=1}^{m}\left|b_{j}\right| \sup _{t \in I}|x(t)-y(t)| \int_{0}^{\eta_{j}} \mathrm{~d} s+k \sup _{t \in I}|x(t)-y(t)| \int_{0}^{t} \mathrm{~d} s \\
& \leq k T|B| \sum_{j=1}^{m}\left|b_{j}\right|\|x-y\|+k T\|x-y\| \leq k T\left(1+|B| \sum_{j=1}^{m}\left|b_{j}\right|\right)\|x-y\| \leq K\|x-y\|
\end{aligned}
$$

and the solution $x$ of the integral Equation (4) is positive for $t \in[a, T]$. This complete the proof.

\section{Local Existence of Solution}

Theorem 3.1. Let $f$ be satisfies the Lipschitz condition. If $T<k\left(1+|B| \sum_{j=1}^{m}\left|b_{j}\right|\right)^{-1}$ then the nonlocal Cauchy problem (1)-(2) has a unique nondecreasing positive solution.

Proof. Define the operator $T: C[0, T] \rightarrow C[0, T]$ by $T x(t)=B\left(x_{1}-\sum_{j=1}^{m} b_{j} \int_{0}^{\eta_{j}} f(s, x(s)) \mathrm{d} s\right)+\int_{0}^{t} f(s, x(s)) \mathrm{d} s$.

Let $x, y \in C[0, T]$, then but if

$$
K=k T\left(1+|B| \sum_{j=1}^{m}\left|b_{j}\right|\right)<1,
$$

then we get

$$
\|T x-T y\| \leq K\|x-y\|,
$$

which proves that the map $T: C[0, T] \rightarrow C[0, T]$ is contraction.

Applying the Banach contraction fixed point theorem we deduce that the integral Equation (4) has a unique solution $x \in C[0, T]$.

To complete the proof, we prove that the integral
Equation (4) satisfies nonlocal problem (1)-(2). Differentiating (4), we get

$$
x^{\prime}(t)=f(t, x(t)) .
$$

Let $t=\eta_{j}$ in (4), we obtain

$$
\begin{aligned}
x\left(\eta_{j}\right)= & B\left\{x_{1}-\sum_{j=1}^{m} b_{j} \int_{0}^{\eta_{j}} f(s, x(s)) \mathrm{d} s\right\} \\
& +\int_{0}^{\eta_{j}} f(s, x(s)) \mathrm{d} s,
\end{aligned}
$$

then

$$
\sum_{j=1}^{m} b_{j} x\left(\eta_{j}\right)=x_{1}
$$


This implies that there exist a unique nondecreasing positive solution $x \in C[0, T]$ of the nonlocal Cauchy problem (1)-(2), This complete the proof.

\section{Continuous Dependence of the Solution}

Consider the nonlocal Cauchy problem

$$
(\tilde{P})\left\{\begin{aligned}
x^{\prime}(t) & =f(t, x(t)), t \in[0, T], \\
\sum_{j=1}^{m} b_{j} x\left(\eta_{j}\right) & =\tilde{x}_{1}, \quad \text { and } \eta_{j} \in(0, a) \subset[0, T] .
\end{aligned}\right.
$$

Definition 4.1. The solution of the nonlocal Cauchy problem (1)-(2) continuously dependence on $x_{1}$ if

$$
\begin{aligned}
& \forall \varepsilon>0, \exists \delta(\varepsilon)>0, \text { such that }\left|x_{1}-\tilde{x}_{1}\right|<\delta, \\
& \text { then }|x(t)-\tilde{x}(t)|<\varepsilon
\end{aligned}
$$

where $\tilde{x}(t)$ is the solution of the nonlocal Cauchy problem $\tilde{P}$.

Now we have the following theorem

Theorem 4.1. The solution of the nonlocal Cauchy problem (1)-(2) continuously dependence on $x_{1}$.

Proof. Let $x(t), \tilde{x}(t)$ are the solutions of (1)-(2) and $\tilde{P}$ respectively.

Then we can get

$$
\begin{gathered}
x(t)-\tilde{x}(t)=B\left(x_{1}-\tilde{x}_{1}\right)-B \sum_{j=1}^{m} b_{j} \int_{0}^{\eta_{j}} f(s, x(s)) \mathrm{d} s+B \sum_{j=1}^{m} b_{j} \int_{0}^{\eta_{j}} f(s, \tilde{x}(s)) \mathrm{d} s \\
+\int_{0}^{t}\{f(s, x(s))-f(s, \tilde{x}(s))\} \mathrm{d} s \\
|x(t)-\tilde{x}(t)| \leq|B|\left|x_{1}-\tilde{x}_{1}\right|+|B| \sum_{j=1}^{m}\left|b_{j}\right| \int_{0}^{\eta_{j}}|f(s, x(s))-f(s, \tilde{x}(s))| \mathrm{d} s+\int_{0}^{t}|f(s, x(s))-f(s, \tilde{x}(s))| \mathrm{d} s \\
\leq|B|\left|x_{1}-\tilde{x}_{1}\right|+k|B| \sum_{j=1}^{m}\left|b_{j}\right| \sup _{t \in I} \int_{0}^{\eta_{j}}|x(s)-\tilde{x}(s)| \mathrm{d} s+k \sup _{t \in I} \int_{0}^{t}|x(s)-\tilde{x}(s)| \mathrm{d} s \\
\leq|B|\left|x_{1}-\tilde{x}_{1}\right|+k|B| \sum_{j=1}^{m}\left|b_{j}\right| \sup _{t \in I}|x(t)-\tilde{x}(t)| \int_{0}^{\eta_{j}} \mathrm{~d} s+k \sup _{t \in I}|x(t)-\tilde{x}(t)| \int_{0}^{t} \mathrm{~d} s \\
\|x-\tilde{x}\| \leq|B|\left|x_{1}-\tilde{x}_{1}\right|+k T|B| \sum_{j=1}^{m}\left|b_{j}\right||| x-\tilde{x}\|+k T\| x-\tilde{x}\left\|\leq|B|\left|x_{1}-\tilde{x}_{1}\right|+k T\left(1+|B| \sum_{j=1}^{m}|b j|\right)\right\| x-\tilde{x} \| \\
\left(1-k T\left(1+|B| \sum_{j=1}^{m}\left|b_{j}\right|\right)\right)|| x-\tilde{x}\left\|\leq|B|\left|x_{1}-\tilde{x}_{1}\right|\right\| x-\tilde{x} \| \leq\left(1-k T\left(1+|B| \sum_{j=1}^{m}\left|b_{j}\right|\right)\right)^{-1}|B|\left|x_{1}-\tilde{x}_{1}\right| .
\end{gathered}
$$

Therefore, for $\delta>0$ such that

we can find

$$
\left|x_{1}-\tilde{x}_{1}\right|<\delta(\varepsilon)
$$

$$
\varepsilon=\left(1-k T\left(1+|B| \sum_{j=1}^{m}\left|b_{j}\right|\right)\right)^{-1}|B| \delta
$$

such that $\|x-\tilde{x}\| \leq \varepsilon$, which complete the proof theorem.

\section{Global Existence of Solution}

Theorem 5.1. Let $f$ be satisfies the Lipschitz condition, then the nonlocal Cauchy problem (1)-(2) has a unique nondecreasing positive solution.

Proof. Define the operator $T: C\left[t_{0}, T\right] \rightarrow C\left[t_{0}, T\right]$ by the Equation (9).

Let $x, y \in C\left[t_{0}, T\right]$, then

$$
\begin{gathered}
T x(t)-T y(t)=-B \sum_{j=1}^{m} b_{j} \int_{0}^{\eta_{j}} f(s, x(s)) \mathrm{d} s+\int_{0}^{t} f(s, x(s)) \mathrm{d} s+B \sum_{j=1}^{m} b j \int_{0}^{\eta_{j}} f(s, y(s)) \mathrm{d} s-\int_{0}^{t} f(s, y(s)) \mathrm{d} s \\
=-B \sum_{j=1}^{m} b_{j} \int_{0}^{\eta_{j}}\{f(s, x(s))-f(s, y(s))\} \mathrm{d} s+\int_{0}^{t}\{f(s, x(s))-f(s, y(s))\} \mathrm{d} s, \\
|T x(t)-T y(t)| \leq k|B| \sum_{j=1}^{m}\left|b_{j}\right| \int_{0}^{t_{0}}|x(s)-y(s)| \mathrm{d} s+k \int_{0}^{t}|x(s)-y(s)| \mathrm{d} s \\
e^{-N\left(t-t_{0}\right)}|T x(t)-T y(t)| \leq k|B| \sum_{j=1}^{m}\left|b_{j}\right| e^{-N\left(t-t_{0}\right)} \int_{0}^{t_{0}}|x(s)-y(s)| \mathrm{d}+k e^{-N\left(t-t_{0}\right)} \int_{0}^{t}|x(s)-y(s)| \mathrm{d} s
\end{gathered}
$$




$$
\begin{aligned}
e^{-N\left(t-t_{0}\right)}|T x(t)-T y(t)| \leq & k|B| \sum_{j=1}^{m}\left|b_{j}\right| \int_{0}^{t_{0}} e^{-N\left(t-t_{0}\right)+N\left(s-t_{0}\right)} e^{-N\left(s-t_{0}\right)}|x(s)-y(s)| \mathrm{d} s \\
& +k \int_{0}^{t} e^{-N\left(t-t_{0}\right)+N\left(s-t_{0}\right)} e^{-N\left(s-t_{0}\right)}|x(s)-y(s)| \mathrm{d} s \\
\leq & k|B| \sum_{j=1}^{m}\left|b_{j}\right|\|x-y\| \int_{0}^{t_{0}} e^{-N(t-s)} \mathrm{d} s+k\|x-y\| \int_{0}^{t} e^{-N(t-s)} \mathrm{d} s \\
\leq & k|B| \sum_{k=1}^{m}|| b_{j} \mid\|x-y\|\left\{\frac{e^{-N\left(t-t_{0}\right)}-e^{-N t}}{N}\right\}+k\|x-y\|\left\{\frac{1-e^{-N t}}{N}\right\} \\
\leq & k \frac{k}{N}\left(|B| \sum_{j=1}^{m}\left|b_{j}\right|\left(e^{-N\left(t-t_{0}\right)}-e^{-N t}\right)+\left(1-e^{-N t}\right)\right)\|x-y\| \leq \frac{k}{N}\left(|B| \sum_{j=1}^{m}\left|b_{j}\right|+1\right)\|x-y\|
\end{aligned}
$$

where

$$
K=\frac{k}{N}\left(|B| \sum_{j=1}^{m}\left|b_{j}\right|+1\right) .
$$

Choose $N$ large enough such that $K<1$, then

$$
\|T x-T y\| \leq K\|x-y\|,
$$

therefor the map $T: C\left[t_{0}, T\right] \rightarrow C\left[t_{0}, T\right]$ is contraction.

Applying the Banach contraction fixed point theorem we deduce that the integral Equation (4) has a unique solution $x \in C\left[t_{0}, T\right]$.

To complete the proof, we prove that the integral Equation (4) satisfies nonlocal problem (1)-(2).

Differentiating (4), we get

$$
x^{\prime}(t)=f(t, x(t)) .
$$

Let $t=\eta_{j}$ in (4), we obtain

$$
\begin{aligned}
x\left(\eta_{j}\right)= & B\left\{x_{1}-\sum_{j=1}^{m} b_{j} \int_{0}^{\eta_{j}} f(s, x(s)) \mathrm{d} s\right\} \\
& +\int_{0}^{\eta_{j}} f(s, x(s)) \mathrm{d} s,
\end{aligned}
$$

then

$$
\begin{gathered}
\sum_{j=1}^{m} b_{j} x\left(\eta_{j}\right)=x_{1} . \\
\tilde{P} \text { respectively. } \\
\text { Then we can get } \\
x(t)-\tilde{x}(t)=B\left(x_{1}-\tilde{x}_{1}\right)-B \sum_{j=1}^{m} b_{j} \int_{0}^{\eta_{j}} f(s, x(s)) \mathrm{d} s+B \sum_{j=1}^{m} b_{j} \int_{0}^{\eta_{j}} f(s, \tilde{x}(s)) \mathrm{d} s+\int_{0}^{t}\{f(s, x(s))-f(s, \tilde{x}(s))\} \mathrm{d} s \\
|x(t)-\tilde{x}(t)| \leq|B|\left|x_{1}-\tilde{x}_{1}\right|+|B| \sum_{j=1}^{m}\left|b_{j}\right| \int_{0}^{\eta_{j}}|f(s, x(s))-f(s, \tilde{x}(s))| \mathrm{d} s+\int_{0}^{t}|f(s, x(s))-f(s, \tilde{x}(s))| \mathrm{d} s \\
\leq|B|\left|x_{1}-\tilde{x}_{1}\right|+k|B| \sum_{j=1}^{m}\left|b_{j}\right| \int_{0}^{t_{0}}|x(s)-\tilde{x}(s)| \mathrm{d} s+k \int_{0}^{t}|x(s)-\tilde{x}(s)| \mathrm{d} s \\
e^{-N\left(t-t_{0}\right)}|x(t)-\tilde{x}(t)| \leq e^{-N\left(t-t_{0}\right)}|B|\left|x_{1}-\tilde{x}_{1}\right|+k|B| \sum_{j=1}^{m}\left|b_{j}\right| \int_{0}^{t} e^{-N\left(t-t_{0}\right)+N\left(s-t_{0}\right)} e^{-N\left(s-t_{0}\right)}|x(t)-\tilde{x}(t)| \mathrm{d} s \\
+k \int_{0}^{t} e^{-N\left(t-t_{0}\right)+N\left(s-t_{0}\right)} e^{-N\left(s-t_{0}\right)}|x(t)-\tilde{x}(t)| \mathrm{d} s
\end{gathered}
$$

Definition 6.1. The solution of the nonlocal Cauchy problem (1)-(2) is uniform stable, if $\forall \varepsilon>0, \exists \delta(\varepsilon)>0$, such that

$$
\left|x_{1}-\tilde{x}_{1}\right|<\delta(\varepsilon) \text {, then }|x(t)-\tilde{x}(t)|<\varepsilon .
$$

where $\tilde{X}(t)$ is the solution of the nonlocal Cauchy problem $\tilde{P}$.

Now we have the following theorem

Theorem 6.1. The solution of the nonlocal Cauchy problem (1)-(2) is uniformly stable.

Proof. Let $x(t), \tilde{x}(t)$ are the solutions of (1)-(2) and 


$$
\begin{aligned}
\|x-\tilde{x}\| & \leq|B|\left|x_{1}-\tilde{x}_{1}\right|+k|B| \sum_{j=1}^{m}\left|b_{j}\right|\|x-\tilde{x}\| \int_{0}^{t_{0}} e^{-N(t-s)} \mathrm{d} s+k\|x-\tilde{x}\| \int_{0}^{t} e^{-N(t-s)} \mathrm{d} s \\
& \leq|B|\left|x_{1}-\tilde{x}_{1}\right|+k|B| \sum_{j=1}^{m}\left|b_{j}\right|\left\|x-\tilde{x} \mid\left\{\frac{e^{-N\left(t-t_{0}\right)}-e^{-N t}}{N}\right\}+k\right\| x-\tilde{x} \|\left\{\frac{1-e^{-N t}}{N}\right\} \\
& \leq|B|\left|x_{1}-\tilde{x}_{1}\right|+\frac{k}{N}\left(|B| \sum_{j=1}^{m}\left|b_{j}\right|\left(e^{-N\left(t-t_{0}\right)}-e^{-N t}\right)+\left(1-e^{-N t}\right)\right)\|x-\tilde{x}\| \\
& \left.\leq|B|\left|x_{1}-\tilde{x}_{1}\right|+\frac{k}{N}\left(|B| \sum_{j=1}^{m}\left|b_{j}\right|+1\right)\|x-\tilde{x}\| \mid\right]^{-1}\left|x_{1}-\tilde{x}_{1}\right|
\end{aligned}
$$

Therefore, $\left|x_{1}-\tilde{x}_{1}\right|<\delta(\varepsilon), \Rightarrow\|x-\tilde{x}\|<\varepsilon$, which complete the proof of theorem.

\section{REFERENCES}

[1] B. Ahmad and J. J. Nieto, "Existence of Solution for Nonlocal Boundary Value Problems of Higher-Order Nonlinear Fractional Differential Equations," Abstract and Applied Analysis, Vol. 2009, 2009, pp. 1-9. doi: $10.1155 / 2009 / 494720$

[2] M. Benchohra, J. J. Nieto and A. Ouahab, "Second-Order Boundary Value Problem with Integral Boundary Conditions," Boundary Value Problems, Vol. 2011, 2011, pp. 1-9. doi:10.1155/2011/260309

[3] A. Boucherif, "First-Order Differential Inclusions with Nonlocal Initial Conditions," Applied Mathematics Letters, Vol. 15, No. 4, 2002, pp. 409-414. doi:10.1016/S0893-9659(01)00151-3

[4] A. Boucherif, "Nonlocal Cauchy Problems for First-Order Multivalued Differential Equations," Electronic Journal of Differential Equations, Vol. 2002, No. 47, 2002, pp. 1-9.

[5] A. Boucherif and R. Precup, "On The Nonlocal Initial Value Problem for First Order Differential Equations," Fixed Point Theory, Vol. 4, No 2, 2003, pp. 205-212.

[6] M. Benchohra, E. P. Gatsori and S. K. Ntouyas, "Existence Results for Seme-Linear Integrodifferential Inclusions with Nonlocal Conditions," Rocky Mountain Journal of Mathematics, Vol. 34, No. 3, 2004, pp. 833-848. doi:10.1216/rmjm/1181069830

[7] Y. K. Chang and J. J. Nieto, "Existence of Solutions for Impulsive Neutral Integro-Differential Inclsions with Nonlocal Initial Conditions via Fractional Operators, Numerical Functional Analysis and Optimization, Vol. 30, No. 34, 2009, pp. 227-244. doi:10.1080/01630560902841146

[8] R. F. Curtain and A. J. Pritchard, "Functional Analysis in Modern Applied Mathematics," Academic Press, New York, 1977.

[9] A. M. A. El-Sayed and Sh. A. Abd El-Salam, "On the Stability of a Fractional-Order Differential Equation with Nonlocal Initial Conditin," Electronic Journal of Differ- ential Equations, Vol. 2009, No. 29, 2008, pp. 1-8.

[10] A. M. A. El-Sayed and Kh. W. Elkadeky, "Caratheodory Theorem for a Nonlocal Problem of the Differential Equation $x^{\prime}=f\left(t, x^{\prime}\right)$," Alexandria Journal of Mathematics, Vol. 1, No. 2, 2010, pp. 8-14.

[11] A. M. A. El-Sayed and Kh. W. Elkadeky, "Solutions of a Class of Nonlocal Problems for the Differential Inclusion $x^{\prime}(t) \in F(t, x(t)), "$ Applied Mathematics and Information Sciences, Vol. 5, No. 2, 2011, pp. 413-421.

[12] A. M. A. El-Sayed, E. M. Hamdallah and Kh. W. Elkadeky, "Solutions of a Class of Deviated-Advanced Nonlocal Problem for the Differential Inclusion

$x^{\prime}(t) \in F(t, x(t)), "$ Abstract and Applied Analysis, Vol. 2011, 2011, pp. 1-9. doi:10.1155/2011/476392

[13] E. Gatsoi, S. K. Ntouyas and Y. G. Sficas, "On a Nonlocal Cauchy Problem for Differential Inclusions," Abstract and Applied Analysis, Vol. 2004, 2004, pp. 425-434. doi:10.1155/S108533750430610X

[14] K. Goebel and W. A. Kirk, "Topics in Metric Fixed Point Theory," Cambridge University Press, Cambridge, 1990. doi:10.1017/CBO9780511526152

[15] A. Lasota and Z. Opial, "An Application of the Kakutani-Ky-Fan Theorem in the Theory of Ordinary Differential Equations," Bull. Acad. Polon. Sci. Ser. Sci. Math. Astoronom. Phys, Vol. 13, 1955, pp. 781-786.

[16] R. Ma, "Existence and Uniqueness of Solutions to FirstOrder Three-Point Boundary Value Problems," Applied Mathematics Letters, Vol. 15, No. 2 2002, pp. 211-216. doi:10.1016/S0893-9659(01)00120-3

[17] S. K. Ntouyas, "Nonlocal Initial and Boundary Value Problems: A Survey," In: A. Canada, P. Drabek and A. Fonda, Eds., Hand Book of Differential Equations, Vol. II Elsevier, New York, 2005.

[18] A. Vlez-Santiago, "Quasi-Linear Boundary Value Problems with Generalized Nonlocal Boundary Conditions, Nonlinear Analysis," Theory, Methods and Applications, Vol. 74, 2011, pp. 4601-4621. doi:10.1016/j.na.2011.03.064 